\title{
The Effect of Companions on the SIM Reference Frame
}

\author{
Christopher S. Jacobs and Slava G. Turyshev \\ Jet Propulsion Labaoratory, California Institute of Technology, \\ Pasadena, CA 91109
}

\begin{abstract}
The Space Interferometry Mission (SIM) is a 10-m Michelson space-based optical interferometer designed for precision astrometry ( $4 \mu \mathrm{as}, 3 \mu \mathrm{as} / \mathrm{yr})$ with better accuracy hoped for over a narrow field of view. It is intended to search for planets and investigate a number of problems in Galactic and extra-galactic astronomy.

The accuracy and stability of SIM's celestial reference frame is subject to degradation over the 5-year mission from the reflex motion induced by massive companions of the objects used to construct the celestial reference frame. We present the results of simulations which show the sensitivity of reference frame accuracy to companions as a function of mass and period. We assume that pre-launch ground surveys will eliminate all objects with RMS radial velocity $>10 \mathrm{~m} / \mathrm{s}$. We further assume that the standard astrometric parameters of position, parallax, and proper motion plus acceleration terms in right ascension and declination will be allowed to absorb reflex motion.
\end{abstract}

\section{Background}

The Space Interferometry Mission (SIM, see e.g. Danner, 2000) is a space-based long-baseline optical interferometer for precision astrometry. One of the primary objectives of the SIM instrument is to determine accurately the directions to a grid of stars, together with their proper motions and parallax, improving a priori knowledge by nearly three orders of magnitude over Hipparcos and one order of magnitude over FAME's planned accuracy (Johnston, 2000). The instrument does not measure directly the angular separation between stars but rather it measures the projection of each star direction vector onto the interferometer baseline vector by measuring the pathlength delay of starlight as it passes through the two arms of the interferometer.

Proposals for SIM grid stars include K-III giant stars at distances of $1 \mathrm{kpc}$, $\mathrm{G}$ dwarfs, and main sequence A stars. The most important criterion, except for the fact that there must be enough objects of a particular type brighter than 12th magnitude, is the absence of astrometric jitter which would hinder the determination of astrometric parameters.

Main sequence $\mathrm{O}$ and $\mathrm{B}$ stars are very young and thus found preferentially in associations located in spiral arms degrading the uniformity of their distribution over the sky. Also, radial velocities of early-type stars cannot be measured very precisely, so that the detection of spectroscopic binaries and radial reflex motions 
would be difficult. The very bright $M$ giants are not considered here because of their variability. G dwarfs must be closer than $100 \mathrm{pc}$ in order to have an apparent magnitude $<12$ thereby scaling up the angular size of reflex motions by an order of magnitude.

\section{Simulated reflex motions}

Thus, for our baseline case we consider K-III giants at a distance of $1 \mathrm{kpc}$. We simulate a distribution of 100,000 companions of these $\approx 1.0$ solar mass stars with the companions distributed over the following ranges of properties (Table $1)$.

Table 1. Binary companions: distribution of properties. $M_{J}$ is the mass of Jupiter.

\begin{tabular}{clrrl} 
Item & Parameter & low Value & high value & units \\
\hline 1 & Mass & 0 & 1050 & $M_{J}$ \\
2 & exclude Brown dwarfs & 10 & 80 & $M_{J}$ \\
3 & Period & 0 & 100 & year \\
4 & Time of periastron & 0 & 1 & cycle \\
5 & Argument of periastron & 0 & 360 & deg \\
6 & Longitude of the Node & 0 & 360 & deg \\
7 & Inclination & 0 & 180 & deg \\
8 & Eccentricity & 0 & 0.99 &
\end{tabular}

Note that if the radial velocity RMS $>10 \mathrm{~m} / \mathrm{s}$ over the five-year mission, the simulated companion was discarded and a new companion was generated. Assuming Keplerian orbits, the parameter space given in Table 1 generates orbit radii from 0 to $27 \mathrm{AU}$ (Fig. 1) and astrometric reflex motions of 0 to 13 mas (Fig. 2). Note: this simulation assumes a "dark" companion. In reality SIM has approximately 10 mas fringe spacing so that both stars will simultaneously influence the fringes. This is a topic for further research.

The $10 \mathrm{~m} / \mathrm{s}$ radial velocity filter re-shaped what was initially a uniform distribution in inclination and period to the distributions shown in (Fig. 3 and 4). The radial velocity test preferentially chooses binary systems that are approximately face-on (inclination $=0$ or $180 \mathrm{deg}$ ). It also preferentially allows long-period systems into the list of reference frame candidates (Fig. 4). Note that our test filters candidate stars based on their RMS radial velocity - not average velocity.

\section{Strategy for handling reflex motion}

We investigated a two-pronged approach to removing these reflex motions from the SIM reference frame ("grid"). First the objects are filtered for radial velocity RMS $<10 \mathrm{~m} / \mathrm{s}$. Since we assume that estimating the full seven Keplerian parameters is too costly in terms of the increased demands on observing time for the grid and the increased complexity of parameter estimation, our second prong was to allow position, proper motion, and acceleration parameters to 


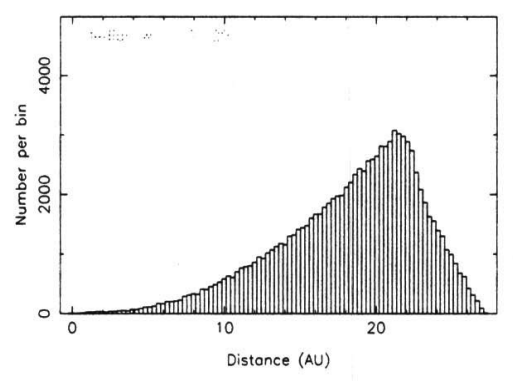

Figure 1. Distribution of orbit radii.

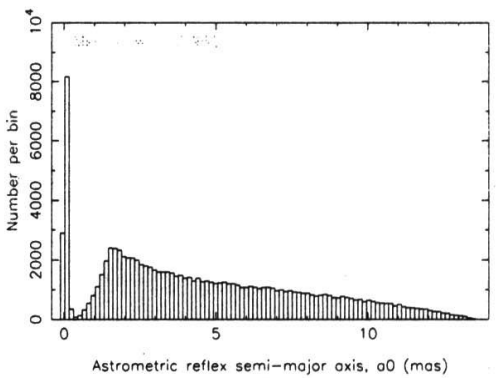

Figure 2. Distribution of astrometric reflex motion semi-axis.

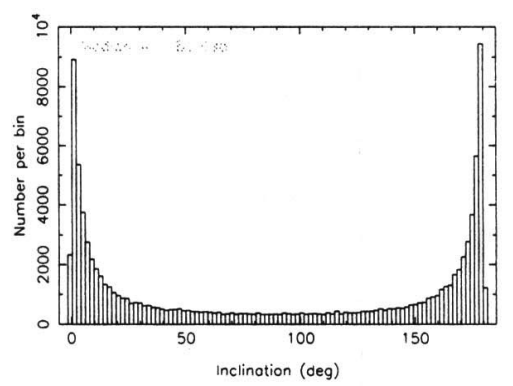

Figure 3. Distribution of inclinations.

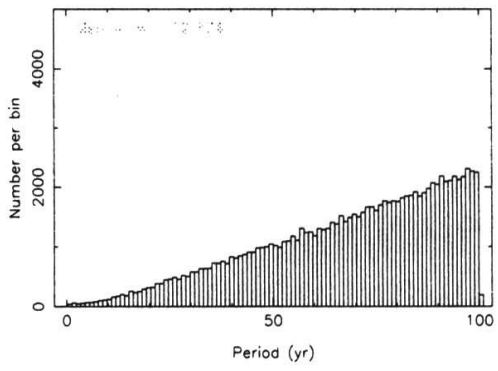

Figure 4. Distribution of periods. 


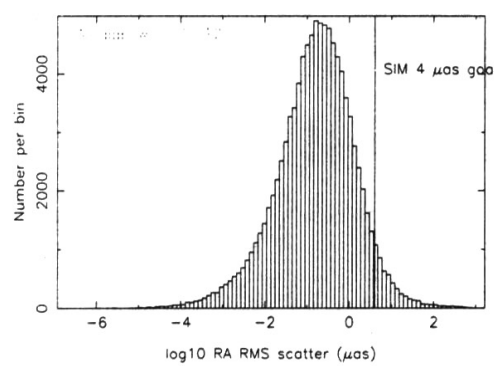

Figure 5. Distribution of RA residuals.

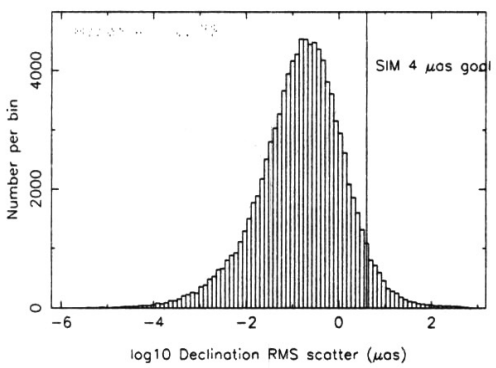

Figure 6. Distribution of Dec residuals.

absorb the Keplerian reflex motion. The position and proper motion parameters were biased on the order of 1 mas and $0.1 \mathrm{mas} / \mathrm{yr}$.

\section{Results}

After estimating these parameters there was a median residual scatter of $\approx$ $0.2 \mu$ as RMS in the instantaneous positions (Fig. $5 \& 6$ ). This is safely below the SIM accuracy goal. Note that estimating acceleration parameters is crucial for modelling long period orbits $(\mathrm{P}>10 \mathrm{yr})$. We also note that there is some prospect for candidates from distances signficantly greater than $1 \mathrm{kpc}$ (Patterson et al., 1999) which would linearly scale down reflex effects as a function of increased distance.

Our simulation calculated instantaneous positions for 100 different observations over the five-year mission for each of 100,000 reference objects. In the absence of a massive companion, the 100 observations per star would be perfectly modeled by the usual five astrometric parameters (position, proper motion, parallax). When the massive companion is introduced, reflex motions are created which result in a scatter about the effective position described by the least-squares adjusted position, proper motion, and acceleration.

Figure 7 shows this scatter vs. mass. The gray-scale contours encode the period. As expected, residuals increase with increasing mass and shorter period. The gap in mass is from our assumption of a brown dwarf desert (10 to 80 Jupiter masses). The SIM accuracy goal of $4 \mu$ as is indicated for comparison.

Figure 8 shows the distribution of these RMS scatters for our 100,000 objects as a function of period. The $x$-axis gives the $\log$ of the period in years and the $y$ axis gives the log of the RMS scatter in mas. The gray-scale variations represent mass contours. As expected, the reflex motion from longer period orbits is more easily absorbed because the reflex motion becomes more linear as the period increases. The placement of the knee in the curve is determined by the 5-year mission lifetime. The two horizontal tails in the distribution of residuals vs. period arise because of the assumed brown dwarf desert. Again the SIM's $4 \mu$ as accuracy goal is indicated for comparison purposes. 


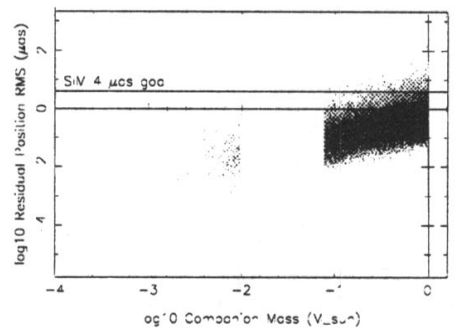

Figure 7. Distribution of position residuals vs. companion mass.

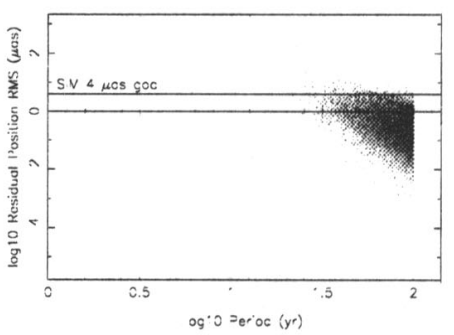

Figure 8. Distribution of Position Residuals vs. Companion Period.

\section{Conclusions}

We have examined the effect of massive companions on the stability of the SIM celestial reference frame. We simulated 1 solar mass stars at $1 \mathrm{kpc}$ having companions with RMS radial velocity $<10 \mathrm{~m} / \mathrm{s}$. If one allows these motions to bias the estimates of position, proper motion and acceleration, the median unmodelled RMS residual would be reduced to below SIM's $4 \mu$ as accuracy goal. We conclude that binary stars - even at $1 \mathrm{kpc}$ - must estimate right ascension and declination accelerations in order to model an acceptably stable reference object.

Looking at separate classes of companions, we note that all but the largest planetary companions should be undetectable or modellable with the addition of RA and Dec acceleration parameters rather than the full 7 Keplerian parameters. We assume a brown dwarf desert from 10 to 80 Jupiter masses and thus ignore this range of companion masses. Stellar binaries with larger masses or shorter periods $(<10 \mathrm{yr}$ ) would benefit from even more stringent radial velocity tests down to $3 \mathrm{~m} / \mathrm{s}$ state-of-the-art accuracy (Butler et al., 1998).

Since SIM's goal is to limit the combination of all errors to $\leq 4 \mu$ as including the contribution from unmodelled binary reflex motion, candidate reference objects should have their radial velocities measured accurately before launch. Such a program would be challenging in that it requires large telescopes and several years of measurements. The best alternative at the present moment for reducing the radial velocity requirements would be to choose reference objects that are farther away. There is evidence that low metallicity $\mathrm{K}$ giants would be observable by SIM at distances of $>2 \mathrm{kpc}$ and perhaps even greater distances (Patterson et al., 1999).

Finally two words of caution: We suspect that at the $\mu$ as level of accuracy many stars may have non-Keplerian signatures in their motions. Secondly, angularly close binaries (< about 10 mas) will interact in the fringe detector, requiring careful simulation. Both topics need further investigation.

Acknowledgments. This work was done at the Jet Propulsion Laboratory, California Institute Technology under contract with NASA. 


\section{References}

Butler, R. P., Marcy, G. W., Williams, E., McCarthy, C., Dosanjh, P., Vogt, S. S., 1996, "Attaining Doppler Precision of 3 M s-1," Pub. Astron. Soc. Pac., 108, 500.

Danner, R., 2000, "Space Interferometer Mission (SIM)," this volume.

Johnston, K., 2000, "Full-sky Astrometric Mapping Explorer (FAME)," this volume.

Patterson, R. J., Majewski, S. R., Kundu, A., Kunkel, W. E., Johnston, K. V., Geisler, D. P., Gieren, W., Muoz, R., 1999, "The Grid Giant Star Survey for the SIM Astrometric Grid," $A A S, 195$, \#46.0.

Velusamy, T., Turyshev, S. G., 1999, 'Microarcsecond Astrometry As A Probe Of Circumstellar Structure,' AAS, 195, \#46.08. 